\title{
ÚLTIMAS ORIENTACIONES DEL TRIBUNAL DE JUSTICIA DE LA UNIÓN EUROPEA SOBRE LA CUESTIÓN PREJUDICIAL
}

\author{
José Antonio Soler MarTíneZ \\ Profesor de Derecho Constitucional \\ y de Derecho Comunitario \\ Universidad Católica San Antonio, Murcia \\ sowieso71@gmail.com
}

\section{INTRODUCCIÓN}

Las cuestiones prejudiciales son uno de los más útiles instrumentos de los poderes judiciales de los Estados miembros de la Unión Europea para hacer valer eficazmente este Derecho en los Estados que integran la Unión. Estas cuestiones prejudiciales son fruto de una duda que tiene el juez nacional sobre la interpretación que merece una disposición de la Unión Europea o incluso sobre su propia validez. Dicha duda surge en el marco de un procedimiento del que está conociendo y en el que entra en juego, de manera decisiva a los efectos de dictar sentencia el ordenamiento de la Unión, bien porque sus disposiciones resultan directamente de aplicación en el asunto, bien porque actúan como parámetro de interpretación del Derecho nacional aplicable. Son pues los jueces nacionales quienes aprecian la necesidad de una decisión del Tribunal de Justicia de la Unión Europea y toman la iniciativa de dirigirse a él por la vía hoy prevista en el Tratado de Funcionamiento de la Unión Europea, suspendiendo mientras tanto la tramitación del litigio.

Si un juez nacional resuelve un litigio al margen del sistema de fuentes y de sus controles normativos y, con ello, lesiona, menoscaba, limita o reduce la efectividad de los derechos que el ordenamiento de la Unión atribuye a las partes en conflicto, no queda más remedio que acudir a la vía reparatoria que suministra el instituto de la responsabilidad patrimonial del Estado-juez. Para evitar estos supuestos de responsabilidad, entre otras cuestiones prácticas, existe la cuestión prejudicial.

Lo que hace el Tribunal de Justicia en este tipo de procesos es en realidad fijar o establecer la norma europea; su resolución tiene un indudable efecto erga omnes, puesto que afectará también a cualquier otro supues- 
to al que haya que aplicar la disposición interpretada. No hay discrepancia alguna a este respecto porque la finalidad de la cuestión prejudicial es, precisamente, la uniformidad en la interpretación y aplicación del ordenamiento de la Unión en todos los Estados miembros, aunque ello se materialice finalmente por muy diferentes órganos judiciales nacionales, a partir de esta opción establecida desde el origen mismo de este Derecho supranacional.

Las resoluciones dictadas con ocasión del planteamiento de una cuestión prejudicial producen efectos ex tunc, que se extienden tanto hacia el pasado como hacia el futuro o, en otros términos, el Derecho de la Unión debería haber sido aplicado interpretado tal y como el Tribunal de Justicia haya señalado en su sentencia; y por lo tanto, en teoría, el Tribunal no baría nada más que poner de relieve un derecho que ya existía antes de la Sentencia. Por esta misma razón, las sentencias dictadas en cuestiones prejudiciales de validez entrañan que el acto invalidado no ha producido nunca efectos.

La importancia de la cuestión prejudicial en el sistema jurídico de la Unión Europea se refleja en el hecho de que, pese a nacer, sobre el papel, como una vía limitada exclusivamente a la interpretación del Derecho comunitario, excluyéndose así, en principio, cualquier pronunciamiento acerca del Derecho de los Estados miembros, ello no ha impedido que, con el paso de los años, se haya convertido no en una más, sino en la primordial vía de control por el Tribunal de Justicia — bien que indirecta- de la compatibilidad de los Derechos nacionales con el Derecho de la Unión. No debe extrañar por ello, habida cuenta del rol esencial que la cuestión prejudicial desempeña en el sistema de la Unión, el hecho mismo de que el Tribunal estime oportuno emitir unas Recomendaciones a los efectos de «orientar a los órganos jurisdiccionales de los Estados miembros sobre la conveniencia de iniciar un procedimiento prejudicial y proporcionarles indicaciones prácticas sobre la forma y los efectos de tal procedimiento» ${ }^{1}$.

Según la primera de las Recomendaciones del Tribunal de Justicia a los órganos jurisdiccionales nacionales, relativas al planteamiento de cuestiones prejudiciales $^{2}$

«La remisión prejudicial es un mecanismo fundamental del Derecho de la Unión Europea, que tiene por objeto proporcionar a los órganos jurisdic-

${ }^{1}$ Cfr. R. Alonso Garcia, «La cuestión prejudicial, piedra angular de la integración europea», en F. BALaguer y E. ARANa (coords.), Libro homenaje al profesor Rafael Barranco Vela, Cizur Menor, Thomson Reuters-Civitas, 2014, p. 13.

${ }^{2}$ Diario Oficial de la UE, Serie C, núm. 338, de 6 de noviembre de 2012. 
cionales de los Estados miembros los medios para que la interpretación y la aplicación de este Derecho sean uniformes en la Unión»³.

Por otro lado, dada la importancia de la cuestión prejudicial en el Derecho de la Unión, resulta trascendente su buen manejo por parte de los jueces y tribunales nacionales; en especial, en aquellos supuestos en los que el planteamiento de la cuestión prejudicial por la jurisdicción ordinaria nacional puede llegar a entrecruzarse con vías incidentales de intervención, en el mismo proceso principal, de la jurisdicción constitucional interna ${ }^{4}$.

Así pues, en la realidad de las cosas, cuando los jueces nacionales que deciden en última instancia consideren necesaria la interpretación de una norma del ordenamiento jurídico de la Unión para decidir el litigio que están llamados a resolver, quedan siempre abocados a dirigirse al Tribunal de Justicia, obligación que se hace extensiva a todos los órganos jurisdiccionales si esa decisión pasa por declarar la invalidez de un acto o de una disposición del derecho derivado de la Unión.

\section{ADMISIBILIDAD DE LA CUESTIÓN PREJUDICIAL: PROCEDIMIENTO Y RECIENTES ORIENTACIONES JURISPRUDENCIALES}

La cuestión prejudicial es competencia exclusiva del Tribunal de Justicia de la Unión Europea. En este sentido, el art. 267 del Tratado de Funcionamiento de la Unión Europea, atribuye al Tribunal de Justicia compe-

3 Se distingue la cuestión prejudicial de la «opinión consultiva» a que se refiere el Protocolo núm. 16, firmado el 2 de octubre de 2013, del Convenio Europeo de Derechos Humanos: aun cuando tanto la una como la otra persiguen concienciar al juez nacional de su condición de juez europeo, la cuestión prejudicial responde esencialmente a la búsqueda de la uniformidad en el funcionamiento del ordenamiento jurídico de la Unión (una norma común, la europea, comúnmente interpretada y aplicada en todos los Estados miembros), mientras que la opinión consultiva está presidida, tal y como expresamente recuerda el Preámbulo mismo del Protocolo núm. 16, por el reformaciones del principio de subsidiariedad, entendido en el sentido de que «la labor de asegurar el respeto de los derechos garantizados por el Convenio descansa, en primer lugar y ante todo, no tanto en el Tribunal europeo como en las autoridades de las Partes Contratantes» (cfr. R. AlONso GARCIA, «La cuestión prejudicial...», op. cit., pp. 10 y ss., y European Inklings, EUi, IV).

${ }^{4}$ Como afirma Alonso García: «La trascendencia del correcto manejo de la cuestión prejudicial, por lo demás, no sólo concierne a jueces y tribunales nacionales, sino también al propio Tribunal de Justicia, el cual, lejos de estar por encima del bien y del mal, también ha incurrido alguna que otra vez en graves deficiencias que han desembocado en no menos graves consecuencias en el sistema de la Unión» (R. ALONSO GARCIA, «La cuestión prejudicial...», op. cit., pp. 22-23). 
tencia para pronunciarse con carácter prejudicial sobre la interpretación de los Tratados y sobre la validez y la interpretación de los actos y normas de derecho derivado. Cuando se plantee en un litigio una cuestión que afecte a la interpretación de unos y otros o a la validez de estos últimos, el juez nacional podrá dirigirse al Tribunal de Justicia para que se pronuncie sobre la misma, si estima necesaria una decisión al respecto para poder emitir su fallo. Por el contrario, si la cuestión se suscita ante un órgano jurisdiccional nacional cuya decisión no sea susceptible de ulterior recurso, queda obligado a plantear la cuestión ${ }^{5}$.

En virtud de lo expuesto, el objeto in genere de la cuestión prejudicial son determinados actos de Derecho de la Unión Europea, diferenciándose:

1) Cuestiones perjudiciales de validez: Su objeto son los actos adoptados por las Instituciones, Órganos y Organismos de la UE, a excepción de los Tratados. En este sentido, el concepto de invalidez del art. 267 TFUE coincide con el de nulidad del art. 263 TFUE.

2) Cuestión prejudicial de interpretación: Su objeto son las normas de Derecho Originario o Derivado; típico o atípico: a) los Tratados, sus anexos, sus protocolos, los Tratados modificativos y los Tratados de adhesión; b) los actos de instituciones, órganos y organismos de la UE, incluidos aquellos que no previstos por los Tratados, como una resolución de carácter no vinculante $\left.{ }^{6} ; c\right)$ las propias Sentencias del TJUE en cuestión prejudicial, ya sea planteada la nueva cuestión por el anterior juez-tribunal nacional o por otro distinto; d) los principios generales del Derecho de la Unión; e) los tratados internacionales concluidos por la UE, en cuanto que forman parte, desde su entrada en vigor, del orden jurídico de la Unión ${ }^{7}$.

5 A la hora de plantear una cuestión prejudicial (ya sea de interpretación o de validez) los órganos jurisdiccionales no sólo toman (y no sólo deben tomar) en consideración las reglas del actual art. 267 TFUE, tal y como han sido interpretadas por el TJUE a partir de sus sentencias en los asuntos Cilfit y Foto-Frost, ni basan su decisión únicamente en una convicción íntima sobre el alcance o sobre la validez de la norma comunitaria considerada. En ocasiones, otros factores vinculados a cuestiones de carácter interno también entran en juego y lo hacen de forma apropiada. Debería, sin embargo, evitarse el recurso prejudicial al Tribunal Europeo por razones que resulten manifiestamente ajenas a la recta aplicación del Derecho de la UE y, desde luego, los jueces de Luxemburgo debieran actuar de forma más estricta en el trámite de admisibilidad cuando les es dado advertir que tal es el caso. Cfr. J. Díez-Hochleitner Rodríguez, «Cuestión prejudicial y política judicial», en R. AlonSO y J. I. UGARTEMENDIA (dirs.), La cuestión prejudicial europea, Oñate, IVAP, 2014, p. 178.

${ }^{6}$ SSTJUE núm. C-113/75, de 15 de junio de 1936, Frecassetti, p. 933, y núm. C-188/91, de 21 de enero de 1993, Deutche Shell, p. 18.

7 Se ha de concluir, por tanto, que en el estado actual de la jurisprudencia del Tribunal de Justicia de la Unión Europea los jueces de los Estados miembros están obligados a dirigirse al 
3) Exclusión de competencia del TJUE: No pueden ser objeto de una cuestión prejudicial: a) cuestiones que no conciernen al Derecho de la UE, en concreto, de derecho puramente interno, cuando todos los elementos del litigio sólo afectan a un Estado miembro; $b$ ) cuestiones sobre hechos anteriores a la adhesión de un Estado miembro a la $\left.\mathrm{UE}^{8} ; c\right)$ sobre tratados internacionales entre Estado miembro o con terceros fuera del marco de la $\mathrm{UE}^{9}$; d) no basta con la mera invocación de la Carta de Derechos Fundamentales de la Unión Europea cuando no se aplica Derecho de la Unión de manera precisa e incondicional ${ }^{10}$.

Constituyendo la cuestión prejudicial un diálogo entre jueces, el Tribunal de Justicia de la Unión Europea está al servicio del juez nacional, quien es soberano respecto del reenvío. De ello se extrae:

1) que las partes en el litigio principal no pueden dirigirse al TJUE directamente; la cuestión prejudicial no es un derecho de las partes (a diferencia del proceso ante el Tribunal Europeo de Derechos Humanos) sino un mecanismo cooperación entre jueces en el que las partes no tienen la última palabra;

2) el juez nacional no está obligado a plantear la cuestión prejudicial por el solo hecho de que se lo soliciten las partes; al contrario, puede plantear la cuestión de oficio; por lo tanto, la apreciación de la pertinencia y necesidad de la cuestión prejudicial corresponde al juez nacional;

3) es el juez nacional quien exclusivamente decide las preguntas a formular (número, orden, contenido y formulación), lo cual no impide que el TJUE pueda reformular dichas preguntas si considera que así resultará más útil para el juez de reenvío, o incluso puede ir más allá de las cuestiones planteadas, sin estar vinculado por las preguntas de las partes;

4) el juez nacional decide unilateralmente el momento procesal oportuno para plantear la cuestión al TJUE, sin que el derecho nacional pueda imponer obstáculos a la aplicación del Derecho de la Unión Europea en el planteamiento de las cuestiones perjudiciales;

5) el juez nacional es dueño de retirar la cuestión prejudicial si — por cualquier razón, correcta o incorrecta — estima que la respuesta del TJUE

Tribunal de Justicia cuando se les suscite una cuestión prejudicial de validez, obligación que únicamente alcanza a los que resuelven en última instancia si la cuestión es de interpretación.

8 STJUE núm. C-168/06, de 6 de marzo de 2007, CerámiKa Paradyz, p. 29.

9 STJUE núm. C-130/73, de 27 de noviembre de 1973, Vandeweghe, p. 1329.2.

10 SSTJUE núm. C-399/11, de 26 de febrero de 2013, Melloni, p. 60, y núm. C-617/10, Ákerberg-Fransson, de 26 de febrero de 2013, p. 29. 
no resulta ya necesaria para la solución del litigio; en esos casos, el TJUE hará constar la solicitud de retirada, sin perjuicio de que pueda postergar su efectividad hasta la fecha del dictado de la sentencia; y,

6) los órganos judiciales superiores nacionales no deben obstaculizar el planteamiento autónomo de cuestiones por los jueces nacionales inferiores; el punto crítico es que el juez nacional inferior puede por vía de la cuestión prejudicial al TJUE soslayar las posiciones de sus tribunales superiores nacionales ${ }^{11}$.

En cuanto a la admisibilidad de la cuestión prejudicial, se puede distinguir la inadmisibilidad «por razón de la naturaleza del litigio o de la cuestión», de la inadmisibilidad «por defectos en el planteamiento de la cuestión prejudicial».

\section{Inadmisibilidad por la naturaleza del litigio o de la cuestión}

La cuestión prejudicial se declara manifiestamente inadmisible ${ }^{12}:$ a) cuando carezca de relación con objeto del litigio principal o sin incidencia para su solución ${ }^{13} ; b$ ) cuando se trate de la cuestión hipotética ya que la función del TJUE no es pronunciarse sobre cuestiones de orden general ni emitir opiniones ${ }^{14}, \mathrm{o} c$ ) cuando el litigio principal presente un carácter ficticio o artificial o haya perdido el objeto ${ }^{15}$.

${ }^{11}$ El planteamiento de la cuestión prejudicial constituye obligación en dos supuestos: a) respecto de las resoluciones de las jurisdicciones nacionales que no son susceptibles de recurso (art. 267 TFUE) (no obstante, el TJUE admite algunas excepciones); $b$ ) por exigencia jurisprudencial, con la finalidad de obligar a todos los jueces nacionales $-\mathrm{y}$ no solamente a los que dictan resoluciones irrecurribles - a plantear cuestiones perjudiciales al TJUE cuando tengan dudas sobre la validez de un acto de la Unión.

12 Arts. 99 y 100 RPTJUE.

13 STJUE núm. C-661/11, de 19 de septiembre de 2013, Martín y Paz Difusion.

${ }_{14}$ STJUE núm. C-149/82, de 3 de febrero de 1983, Robards, p. 19.

15 STJUE núm. C-104/79, de 11 de marzo de 1980, Foglia/Novello; SSTJU núm. C-189/13, de 13 de julio de 2014, Da Silva, p. 29, y núm. C-180/12, de 24 de octubre de 2013, Stoilov $i$ Ko, y STJUE núm. C-600/13, de 30 de abril de 2014, Intelcom Services Ltd. Las causas de inadmisibilidad deben ser manifiestas. A contrario, las cuestiones planteadas al TJUE ostentan una «presunción de pertinencia». Si a ello añadimos que el Tribunal no puede, en el ámbito de la cuestión prejudicial, ni establecer ni apreciar los hechos del litigio principal (STJUE núm. C-51/74, de 23 de enero de 1975, Hulst, p. 12), se concluye que el TJUE se muestra receptivo, efectuando interpretación extensiva pro quaestione. 


\section{Inadmisibilidad por inadecuado planteamiento de la cuestión}

El art. 94 RPTJUE (y el p. 22 de las Recomendaciones 2012/C 338/01) ${ }^{16}$ exige que la demanda prejudicial contenga ${ }^{17}: a$ ) elementos fáctico- normativos: el objeto y los hechos pertinentes, o datos fácticos; las disposiciones y jurisprudencia nacional susceptibles de ser aplicadas; $b$ ) fundamentos jurídicos: los razonamientos sobre el enlace entre el Derecho la UE en la legislación nacional que justifican la pregunta, y c) la(s) pregunta(s). Estos contenidos de la demanda prejudicial son de escrupuloso cumplimiento, pudiendo la cuestión prejudicial ser declarada inadmisible por la forma defectuosa en que sea planteada ${ }^{18}$.

Por tanto, corresponde a la jurisdicción de reenvío definir mínimamente el cuadro fáctico de la cuestión así como el marco jurídico que la justifica. Estos elementos son imprescindibles no sólo por mor de la admisibilidad de la cuestión, sino también por razón de la efectividad del derecho de alegación y tutela judicial de otros intervinientes y Estado miembro, a quienes se les debe dar traslado de la cuestión ${ }^{19}$. El Tribunal de Justicia de la Unión Europea puede rechazar la cuestión como inadmisible, por falta de precisión suficiente al respecto, lo cual no es óbice para que la jurisdicción de reenvío plantee — de nuevo- una segunda cuestión, sobre el mismo objeto, correctamente formulada ${ }^{20}$.

16 Exposición sucinta del objeto del litigio y de los hechos pertinentes haciendo constar datos fácticos; texto de las disposiciones nacionales aplicables y de la jurisprudencia nacional pertinente; razonamientos que justifican la pregunta y su relación con la normativa aplicable; identificación precisa de las disposiciones del Derecho la Unión, y, discrecionalmente, punto de vista del juez a quo sobre la solución a adoptar. Las preguntas deben ser claras, pudiendo figurar al inicio o al final de la resolución de planteamiento.

Otros requisitos formales son: modelo de resolución judicial, la mecanografía, estructuración en apartados, fecha y firma, remisión por correo certificado, dirección electrónica y fax, etcétera.

17 Estos elementos se recogían en la jurisprudencia STJUE núms. C-320/90, C-321/90 y C-322/90, de 26 de enero de 1993, Telemarsicabruzzo y otros, p. 6, o en AaTJUE núm. C-157/92, de 19 de marzo de 1993, Banchero, p. U, y núm. C-167/94, de 7 de abril de 1995, Grau Gomis y otros, p. 9, y que fueron integrado en la reforma del RPTJUE de 29 de septiembre de 2012.

18 ATJUE núm. C-19/14, de 3 de julio de 2014, Talasca, p. 21.

19 STJUE núm. C-370/12, de 27 de noviembre de 2012, Pringle, p. 85.

20 SSTJUE núm. C-234/12, Sky Italia; núm. C-113/13, de 11 de diciembre de 2014, Azienda Sanitaria Locale núm. 5 «Specino» y otros; núm. C-470/13, de 18 de diciembre de 2014, Generalli Providenzia Biztositó, y ATJUE 3D I, núm. C-107/14, de 17 de julio de 2014. 
Por otra parte, además, el TJUE se declara incompetente sobre las cuestiones prejudiciales relativas a la compatibilidad del derecho nacional (o de una práctica nacional) con el Derecho de la Unión; sobre las cuestiones prejudiciales que versen sobre la interpretación del derecho nacional, en casos de planteamiento de varias cuestiones, puede declarar inadmisibles por esta causa algunas, y, asimismo, declarar inadmisibles las preguntas sobre el alcance de un texto jurídico que no ha sido adoptado por una institución, órgano u organismo de la $\mathrm{UE}^{21}$.

Por lo que se refiere a la tramitación ante el Tribunal Europeo de Justicia (ad quem), este órgano judicial, en el tratamiento de las cuestiones perjudiciales, es muy flexible y poco formalista ${ }^{22}$. La flexibilidad del procedimiento es una de las características de la cuestión prejudicial, producto de que no se trata de un recurso sino de un dialogo o intercambio entre tribunales, en el que el papel del TJUE consiste en ayudar al juez nacional a llevar a cabo la aplicación del Derecho la Unión en el litigio que le sometido en origen. Así el Tribunal es libre de: 1) modificar el orden de las cuestiones planteadas, si ello le parece más lógico (o más pedagógico); 2) reagrupar las cuestiones, o no responder a algunas de ellas, si esto le parece necesario; 3) reformular las cuestiones planteadas, si ello puede resultar de utilidad al juez nacional; 4) ir más allá de las cuestiones planteadas, si el TJUE considera que el juez nacional necesita, para la solución del litigio sometido, ser esclarecido sobre un punto que no ha sido suscitado en su reenvío judicial.

Así, el TJUE puede dar su interpretación respecto de textos no citados por el juez a quo, y responder a cuestiones no formuladas. Incluso el Tribunal ha llegado a reformular una cuestión prejudicial de interpretación, considerándola como una cuestión prejudicial de validez $z^{23}$.

Los efectos de las sentencias dictadas por el TJUE sobre una cuestión prejudicial están revestidas de la autoridad de cosa juzgada y se imponen

${ }^{21}$ En la más reciente jurisprudencia se puede ver ATJUE núm. C-608/14, de 7 de mayo de 2015, Pondiche. No obstante, el TJUE no está vinculado por la literalidad de la cuestión prejudicial planteada, y respecto de una cuestión sobre la compatibilidad del Derecho nacional con el Derecho la Unión, ha sentado que «nada impide al Tribunal dar una respuesta útil a la jurisdicción de reenvío, facilitándole los elementos de interpretación relevantes del Derecho de la Unión» (STJUE núm. C-25/11, de 16 de febrero de 2012, Varzim Sol). Por lo demás, el TJUE puede igualmente proceder a la interpretación de las disposiciones del Derecho de la Unión que no han sido expresamente mencionadas en las cuestiones perjudiciales planteadas.

22 Se rige por el título III del Reglamento de Procedimiento del TJUE (arts. 93 a 118).

23 STJUE núm. C-145/79, de 15 de octubre de 1980, Roquete Frères. 
frente a todos. Así resulta - en concreto- respecto de la interpretación del Derecho de la Unión dada por el Tribunal, la cual vincula, no sólo a la jurisdicción de reenvío, sino a las jurisdicciones de todos los Estados miembros, a sus Gobiernos y Administraciones, así como a las Instituciones de la Unión, que deben - desde ése momento- aplicar e interpretar el Derecho de la UE conforme a lo que el TJUE haya juzgado.

$\mathrm{Si}$ se trata de una sentencia sobre cuestión prejudicial de validez, que anula un acto, el juez nacional se debe abstener de aplicar dicha norma, al igual que las Instituciones de la UE, que debe extraer las consecuencias de dicha declaración de invalidez, y las autoridades nacionales, las cuales deben extraer las consecuencias respecto de las medidas de ejecución nacionales necesarias. Si la sentencia no anula el acto, el mismo continúa aplicándose pero — como hemos visto-, nada impide al órgano jurisdiccional nacional volver plantear al TJUE de nuevo una cuestión prejudicial de validez, si bien por motivos diferentes a los anteriormente planteados.

Finalmente, hay que recordar que Europa ha sufrido una crisis de deuda soberana. Para superarla, la Unión y los Estados miembros recurren a medidas en parte poco convencionales. En este sentido, se deben citar dos de las últimas sentencias del Tribunal de Justicia de la Unión Europea, de gran complejidad técnica y jurídica, que decidieron sobre distintas cuestiones prejudiciales planteadas ante dicho Alto Tribunal, referentes al caso Pringle (núm. C-370/12) a instancia de la Corte Suprema de Irlanda, que dio lugar a la sentencia de fecha 27 de noviembre de 2012, del Pleno del Tribunal ${ }^{24}$, que, entre otras cuestiones, determinó que «no se ha revelado ningún aspecto que pueda afectar a la validez de la Decisión 2011/199/UE del Consejo Europeo, de 25 de marzo de 2011, que modifica el art. 136 del Tratado de Funcionamiento de la Unión Europea ${ }^{25}$ en relación con un mecanismo de estabilidad para los Estados miembros cuya moneda es el euro (MEDE)».

Esta decisión judicial constituye una respuesta directa de la Unión Europea y es una consecuencia de la leal cooperación respecto de la manera de afrontar la crisis financiera haciendo cambios constitucionales europeos, articulado como recurso judicial.

24 bttp://curia.europa.eu/juris/document/document.jsf?docid=130381Edoclang=ES.

25 El apartado 3 del art. 136 TFUE es el que contiene la regla de habilitación según la cual: «Los Estados miembros cuya moneda es el euro podrán establecer un mecanismo de estabilidad que se activará cuando sea indispensable para salvaguardar la estabilidad de la zona del euro en su conjunto. La concesión de toda ayuda financiera necesaria con arreglo al mecanismo se supeditará a condiciones estrictas». 
Por otra parte, el Tribunal Constitucional Federal de Alemania, con fecha 14 de enero de 2014, interpuso ante el Tribunal de Justicia de la Unión Europea, petición de decisión prejudicial, actuando como recurrente el Sr. Gauweiler y otros (Asunto núm. C-62/14).

En la sentencia de la Gran Sala, de fecha 16 de junio de 2015, en el recurso que tiene por objeto la petición de decisión prejudicial referente a la validez de los acuerdos del Consejo de Gobierno del Banco Central Europeo, de 6 de septiembre de 2012, sobre ciertas características técnicas de las operaciones monetarias de compraventa de títulos («Outright Monetary Transactions», «OMT»), por parte del Eurosistema en los mercados secundarios de deuda soberana, y la interpretación de determinados artículos del Tratado Fundacional de la Unión Europea ${ }^{26}$, el Tribunal de Justicia decidió que los artículos cuestionados referentes a los Estatutos del Sistema Europeo de Bancos Centrales y del Banco Central Europeo, deben interpretarse en el sentido de que autorizan al Sistema Europeo de Bancos Centrales a adoptar un programa de compra de bonos soberanos en los mercados secundarios, en las condiciones que se determinan. De este modo, el Tribunal concede al Banco Central Europeo un amplio margen de discreción para la ejecución de la política monetaria.

26 Arts. 119, 123 y 127 TFUE, y arts. 17 a 24 del Protocolo (núm. 4) sobre los Estatutos del Sistema Europeo de Bancos Centrales. 